\title{
Translocation of rigid rod-shaped virus through various solid-state nanopores
}

Hongwen $\mathrm{Wu}^{1}$, Yuhao Chen ${ }^{2}$, Qizhao Zhou ${ }^{3}$, Rongliang Wang ${ }^{1}$, Baicheng $\mathrm{Xia}^{2}$, Dejun $\mathrm{Ma}^{4}$, Kaifu Luo ${ }^{2}$, Quanjun Liu ${ }^{*}$.

${ }^{1}$ State Key Laboratory of Bioelectronics, Southeast University, Nanjing, 210096, China.

${ }^{2}$ CAS Key Laboratory of Soft Matter Chemistry, and Department of Polymer Science and Engineering, University of Science and Technology of China, Hefei, 230026, China.

${ }^{3}$ The Third Affiliated Hospital of Southern Medical University, Guangzhou, 510000, China.

${ }^{4}$ State Key Laboratory of Elemento-Organic Chemistry and Department of Chemical Biology, National Pesticide Engineering Research Center (Tianjin), Nankai University, Tianjin, 30007

1, China.

*1qj@seu.edu.cn

\section{Table of content}

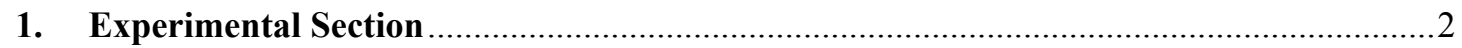

Nanopore fabrication and data acquisition .............................................................

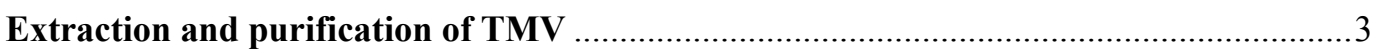

2. Langevin dynamics simulation of TMV translocations .................................................

3. Figure S2. DLS measurement of TMV dispersed in $1 \mathrm{M} \mathrm{NaCl}$ (PH 7.0) ...........................

4. Figure S3. Current trace of TMV getting stuck in the pore .........................................

5. The study of the conductance blockage of the vertical translocation $\left(\Delta G_{0}\right)$ and the maximum conductance blockage $\left(\Delta \boldsymbol{G}_{\max }\right)$ as a function of pore diameter.............................. 8

6. Figure S8. The current traces of TMV translocation through $25 \mathrm{~nm}$ nanopore at $450 \mathrm{mV}$,

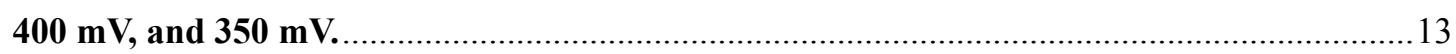

7. Figure S9. The current traces of TMV translocation through $50 \mathrm{~nm}$ nanopore at $200 \mathrm{mV}$,

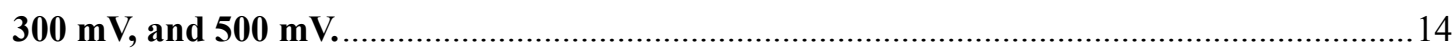

8. Figure S10. The model and simulated distribution of EOF in a $40 \mathbf{~ n m}$ nanopore ............15

9. Figure S11. The simulated conductance change of the vertical translocation $\left(\Delta G_{0}\right)$ normalized by open-pore conductance $\left(G_{0}\right)$ as a function of pore diameter...........................16

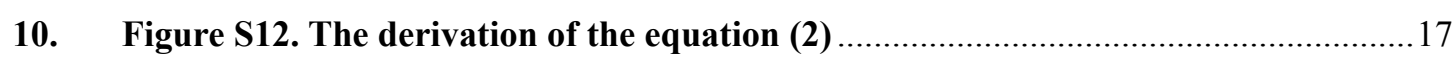

11. Figure S13: The scatter plots of $\Delta I_{\max }$ vs $\Delta I_{\text {end }}$ of the three nanopores at all voltages. 18

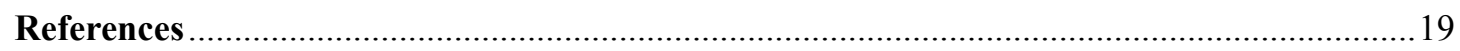




\section{Experimental Section}

\section{Nanopore fabrication and data acquisition}

The silicon chips supporting $30 \mathrm{~nm}$ thick free-standing SiN membranes (DTF-030523, DuraSiN ${ }^{\mathrm{TM}}$ ) were purchased from Protochips, Inc. A FEI Titan 80-300 TEM was used to fabricate and image the nanopores according to the previous report. ${ }^{1}$ Nanopores with diameter larger than $30 \mathrm{~nm}$ were drilled by a Helium Ion Microscopy (HIM) system, and the diameters of the pores were determined by the open-pore conductance. ${ }^{2,3}$ Prior to use, the nanopore containing chips were cleaned in fresh prepared piranha solution at $80{ }^{\circ} \mathrm{C}$ for $30 \mathrm{~min}$, and then washed by DI water. The adsorbed water on the surface of the chip was carefully blotted using a clean Kimwipes (KIMTECH). Then, the chip was assembled in a custom-built Teflon cell with two Viton o-rings to separate the two side of chip, forming two reservoirs. Two Teflon tubing (inner diameter, $0.75 \mathrm{~mm}$ ) were then inserted to the reservoirs respectively to create channels from adding solution, as shown in figure 1a. $1 \mathrm{~mL}$ Syringe connected to 360 $\mu \mathrm{m}$ outside diameter (OD) capillary tubing by a Luer-to-MicroTight Adapter (Upchurch Scientific) was used to extrude the bubbles and add samples, as shown in figure S1. Solutions could be easily changed by flowing liquid through the $360 \mu \mathrm{m}$ OD capillary tubing. After addition, $\mathrm{Ag} / \mathrm{AgCl}$ electrodes were inserted in the two Teflon tubing respectively and connected to a current amplifier (Axopatch 200B, Molecular Devices). By applying a bias voltage over the two electrodes, the negatively charged virus were driven through the pore. The corresponding current was filtered at $10 \mathrm{kHz}$ by a low-pass filter embedded in the amplifier and digitized at $100 \mathrm{kHz}$ or higher (Axon 1440A digitizer, Molecular Devices). Each event was copied and saved as a text file by Clampfit (Molecular Devices), and then a custom Matlab routine was used to analysis the event.
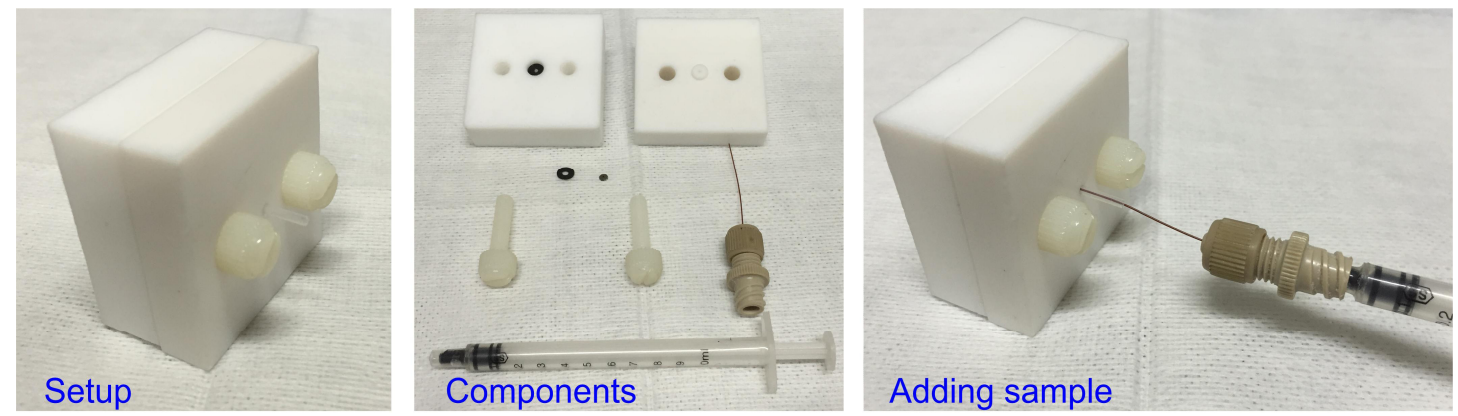

Figure S1. The setup and the components used in our experiments. 


\section{Extraction and purification of TMV}

TMV was received from Long's group according to the literature method. ${ }^{4}$ The $25 \mathrm{~g}$ infected tobacco/soybean leaves were ground into powder with the pestle in the precooled mortar, and then homogenized on ice with $25 \mathrm{~mL} 0.2 \mathrm{M}$ phosphorous salt buffer $(\mathrm{pH} 7.0)$ and $250 \mu \mathrm{L}$ mercaptoethanol (1\%). The leaf homogenate liquid was infiltrated into two-layer nylon cloth and collected with another new sterilized tube, followed by stirring with $8 \%$ n-butyl alcohol up to 15 min for complete chlorophyll condensation on the magnetic stirring apparatus. The light yellow supernatant was collected by refrigerated centrifugation at 10,000 g for $20 \mathrm{~min}$ and stirred with $4 \% \mathrm{NaCl}(1 \mathrm{~g})$ and $4 \%$ PEG $6000(1 \mathrm{~g})$ for $6 \mathrm{~h}$ on ice to aggregate virus particles. After centrifugating at 10,000 g for $20 \mathrm{~min}$ and removing the liquid supernatant, the precipate was resuspened and further stirred with $5 \mathrm{~mL} 0.01 \mathrm{M}$ phosphorous salt buffer $(\mathrm{pH}$ 7.0) for $2 \mathrm{~h}$ to be dissolved fully. The suspension was centrifugated at 10,000 g for $20 \mathrm{~min}$ to collect the supernatant. The supernatant was then stirred again with $4 \% \mathrm{NaCl}(0.2 \mathrm{~g})$ and $4 \%$ PEG $6000(0.2 \mathrm{~g})$ overnight on ice to precipitate fully. The precipate was collected by centrifugating at $10,000 \mathrm{~g}$ for $20 \mathrm{~min}$ and stirred again with $2 \mathrm{~mL} 0.01 \mathrm{M}$ phosphorous salt buffer ( $\mathrm{pH}$ 7.0) on ice for $6 \mathrm{~h}$. After centrifugating at 10,000 $\mathrm{g}$ for $5 \mathrm{~min}$, the supernatant was pelleted into the newly-sterilized collection tube as the final virus suspension. The virus concentration was determined at $\mathrm{A}_{260}$ on spectrophotometer and calculated according to the formula $\mathrm{C}(\mathrm{mg} / \mathrm{mL})=\mathrm{A}_{260} / 3.1$. The virus purity was evaluated according to the double parameter ratio $\mathrm{A}_{280} / \mathrm{A}_{260}(0.84)$ and $\mathrm{A}_{260} / \mathrm{A}_{248}(1.09)$. The purified virus suspension was stored at $4^{\circ} \mathrm{C}$ for futher test. 


\section{Langevin dynamics simulation of TMV translocations}

In our simulations, Tobacco mosaic virus was modeled as a coarse-grained bead-spring chain consisting of 17 monomers, ${ }^{5-8}$ with consecutive beads attached to each other by a Finite Extension Nonlinear Elastic (FENE) potential:

$$
U_{F E N E}(r)=-\frac{1}{2} k R_{0}^{2} \ln \left(1-\frac{r^{2}}{R_{0}^{2}}\right)
$$

where $r$ is the distance between consecutive beads. We set the spring constant $k$ to $30 \varepsilon_{0} / \sigma^{2}$, and the maximum allowed separation between connected beads $R_{0}$ to $1.5 \sigma$. Then we employed a shorted-range repulsive Lennard-Jones (LJ) potential to incorporate the excluded volume interaction between beads:

$$
U_{L J}(r)=4 \varepsilon_{0}\left[\left(\frac{\sigma}{r}\right)^{12}-\left(\frac{\sigma}{r}\right)^{6}\right]+\varepsilon_{0}
$$

for $r \leq 2^{1 / 6} \sigma$ and 0 for $r \geq 2^{1 / 6} \sigma$. Here, $\sigma$ is the diameter of a bead, $\varepsilon_{0}$ is the depth of the potential, and $r$ is the distance between a pair of beads. In this work, the size of the bead corresponded to the diameter of TMV, so we chosen $\sigma=18 \mathrm{~nm}$ and the bead mass $m_{0}=$ $4.0 \times 10^{-8} \mathrm{~g}$. The stiffness of the chain was introduced by applying a bending potential between successive bonds:

$$
U_{\text {bend }}(\theta)=\frac{\kappa}{2}\left(\theta-\theta_{0}\right)^{2}
$$

Where $\theta$ is the angle between adjacent bond vectors, the equilibrium value of the bond angle $\theta_{0}$ was set to be $\pi$, and $\kappa$ is the bending constant. To model TMV, we set $\kappa=212$, yielding a persistence length of $170 \sigma$ which is ten times the contour length of TMV.

A continuous surface with thickness $5 / 3 \sigma$ was used to model the membrane in three dimension, and diameter of the cylindrical nanopore was $5 / 3 \sigma$, corresponding to the typical experiment in this work where $d_{\text {pore }}=30 \mathrm{~nm}$ and $l_{\text {pore }}=30 \mathrm{~nm}$. The electric field of the outside of this pore was modeled by ${ }^{9}$

$$
E(r)=\frac{V}{\frac{\pi R}{2}+l_{\text {pore }}} \frac{R^{2}}{2 r^{2}}
$$

while the electric field in the pore was modeled by $V / l_{\text {pore. }} R$ is the pore radius, $V$ is the applied voltage. In Langevin dynamics simulation, each mobile particle was subjected to conservative, frictional, external electric force, and random forces, respectively. The final equation is

$$
m_{i} \ddot{\mathbf{r}}_{i}=\mathbf{F}_{e x t}-\nabla\left(U_{L J}+U_{F E N E}\right)-\xi \mathbf{v}_{i}+\mathbf{F}_{i}^{R}
$$

where $m_{i}$ is the particle's mass, $\xi$ is the frictional coefficient, $\mathrm{v}_{i}$ is the particle's velocity. $\mathrm{F}_{\text {ext }}$ is the applied external electric force, and $\mathbf{F}_{i}^{R}$ is the random force which satisfies the 
fluctuation-dissipation theorem ${ }^{10}$

$$
\begin{gathered}
\left\langle\mathbf{F}_{i}^{R}(t)\right\rangle=0 \\
\left\langle\mathbf{F}_{i}^{R}(t) \cdot \mathbf{F}_{j}^{R}\left(t^{\prime}\right)\right\rangle=2 d k_{B} T \xi \delta_{i j} \delta\left(t-t^{\prime}\right)
\end{gathered}
$$

Here $d$ is the space dimensionality, $k_{B}$ is Boltzmann's constant, $\delta_{\mathrm{ij}}$ is the Kronecker delta, $\delta\left(t-t^{\prime}\right)$ is the Dirac delta function, and $T$ is the absolute solvent temperature. In this work, we used the LJ parameters $\varepsilon_{0}, \sigma$, and monomer's mass $m_{0}$ to fix the system energy, length and mass scales, respectively. Then, the corresponding time scale was given by $t_{L J}=\left(m_{0} \sigma^{2} / \varepsilon_{0}\right)^{1 / 2}$. We set $k_{B} T=0.2 \varepsilon_{0}$, which means the interaction energy $\varepsilon_{0}$ is $2.034 \times 10^{-20} \mathrm{~J}$ at actual temperature $295 \mathrm{~K}$. This leads to a time scale of $7.98 \mathrm{~ns}$. We set the friction coefficient $\xi=$ $147 / t_{L J}$. Thus, the simulation diffusion coefficient $D_{\text {sim,\| }}$ of TMV could be consistent with the experimental one $D_{0, \|}=2.8 \times 10^{-8} \mathrm{~cm}^{2} \mathrm{~s}^{-1} \cdot{ }^{11}$

The applied external force $\mathrm{F}_{\text {ext }}$ was adjusted by matching the Péclet number in experiments and simulations, which was defined as $P \dot{e}=L v / D=\left(\left(L\left\langle\mu_{T M V, p o r e}\right\rangle\right) /\left(D_{0, \|}\right)\right) V^{5}$. We measured the experimental mobility of TMV translocations to be $\left\langle\mu_{T M V \text {, pore }}\right\rangle \approx 24$ $\mathrm{mmV}^{-1} \mathrm{~s}^{-1}$. Based on the known length of TMV and its diffusion coefficient $D_{0, \|}=2.8 \times 10^{-8} \mathrm{~cm}^{2} \mathrm{~s}^{-1}$, we obtained $P e_{e x p} \approx 240$ for $V=100 \mathrm{mV}$. Then, we measured the diffusion coefficient of TMV in simulations to be $D_{s i m, \|}=7.5 \times 10^{-5} \sigma^{2} / t_{L J}$. Assuming that the simulation Péclet number is $P \dot{e}_{s i m} \approx 240$, we found the applied external force should be $\mathrm{F}_{\text {ext }}$ $\approx 1.9 \varepsilon_{0} / \sigma$. The Langevin equation was then integrated in time by a method described by Ermak and Buckholz. ${ }^{12}$ 


\section{Figure S2. DLS measurement of TMV dispersed in $1 \mathrm{M} \mathrm{NaCl} \mathrm{(PH} \mathrm{7.0)}$}

DLS measurement of TMV dispersed in $1 \mathrm{M} \mathrm{NaCl}$ ( $\mathrm{PH} 7.0$ ) showing a uniform size distribution, and zeta potential was measured to be $-8.92 \mathrm{mV}$ in the same solution.

DLS is used primarily in the analysis of globular particles, it obtains the particles size by measuring the diffusion coefficient of the particle, and the measured size is always called "hydrodynamic" size. However, since the software does not account for the rod-shape of the particle which affects the diffusion coefficient of the particle, quantitative size distribution of rod-shaped particles cannot be obtained. ${ }^{13}$ The average size of $\sim 100 \mathrm{~nm}$ was attributed to the rod shape of TMV, $300 \mathrm{~nm}$ in length, $18 \mathrm{~nm}$ in diameter. It should be note that DLS instrument used here has a fixed detection angle, and it can not be directly used to characterize the size of rod-shaped particles. Traditionally, DLS was used to measure the translational and rotational diffusion coefficients which can indirectly confirm the size and shape of TMV. ${ }^{14,15}$ Because TMV is a rigid rod, there were translational and rotational diffusions when it was placed in DLS measurement, thus the average size of TMV was usually measured to be $70-140 \mathrm{~nm}$ by DLS instrument with a fixed detection angle. ${ }^{16-19}$ The difference of the average size could be attributed to the solution condition such as the kind of ions, the salt concentration, $\mathrm{PH}, \mathrm{TMV}$ concentration and so on, and the $\sim 100 \mathrm{~nm}$ average size in the DLS measurement here is consistent with the previous literatures, indicating no aggregation of TMV.

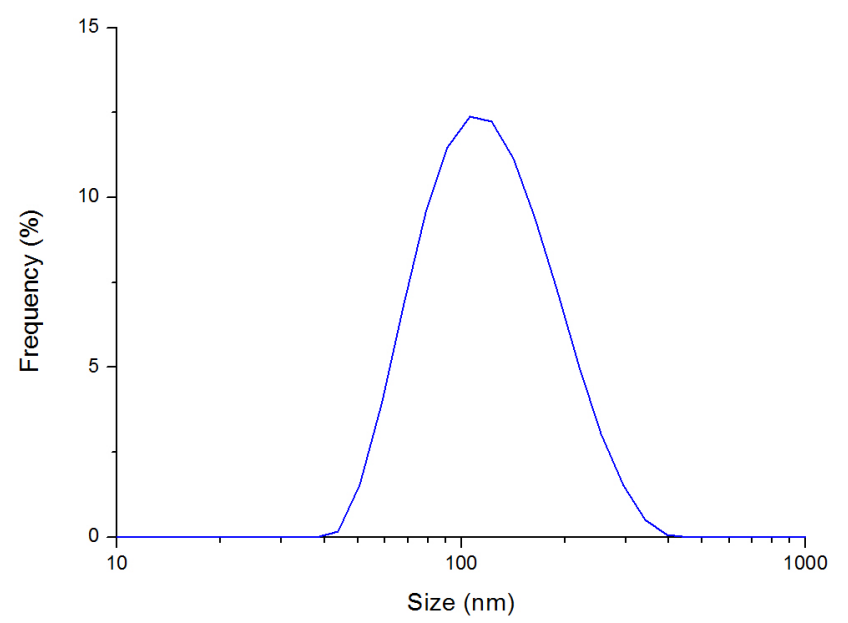




\section{Figure S3. Current trace of TMV getting stuck in the pore}

The current trace showing that TMV get stuck in the pore which can be clean out by reversing the applied voltage. The black arrow points at the place where the voltage is reversed.

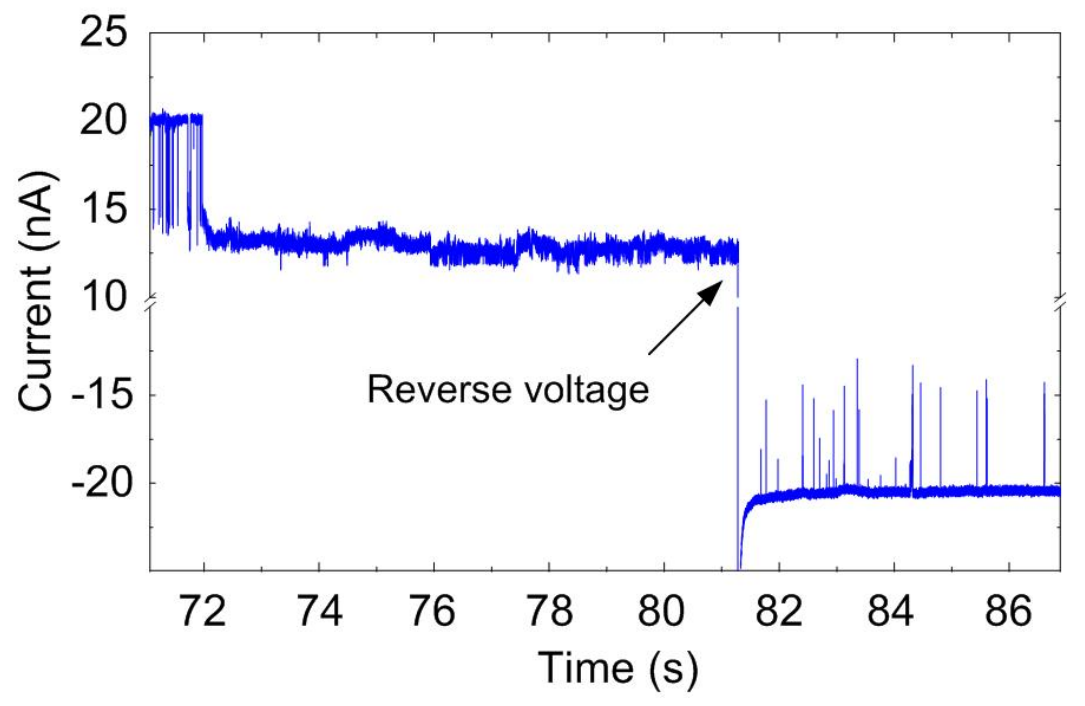


5. The study of the conductance blockage of the vertical translocation $\left(\Delta G_{0}\right)$ and the maximum conductance blockage ( $\left.\Delta G_{\max }\right)$ as a function of pore diameter.

Besides $30 \mathrm{~nm}$ nanopore, two additional nanopores with diameter $25 \mathrm{~nm}$ and $50 \mathrm{~nm}$ were used. The current-voltage (I-V) curves at $1 \mathrm{M} \mathrm{NaCl}(\mathrm{PH} 7.0)$ are shown in figure S4a, and the solid lines are the linear fitting of each curve, yielding the conductance of the pores. The insert are the TEM images of $25 \mathrm{~nm}$ (red) and $30 \mathrm{~nm}$ (black) nanopores. However, as the 50 $\mathrm{nm}$ nanopore was fabricated by HIM, it is hard to find it in TEM, and the diameter was calculated by the open-pore conductance $\left(G_{0}\right){ }^{2}$ Figure $\mathrm{S} 4 \mathrm{~b}$ are the representive conductance traces for each pore size. The traces of $25 \mathrm{~nm}$ and $50 \mathrm{~nm}$ pore under the other applied voltages are shown in the following sections.

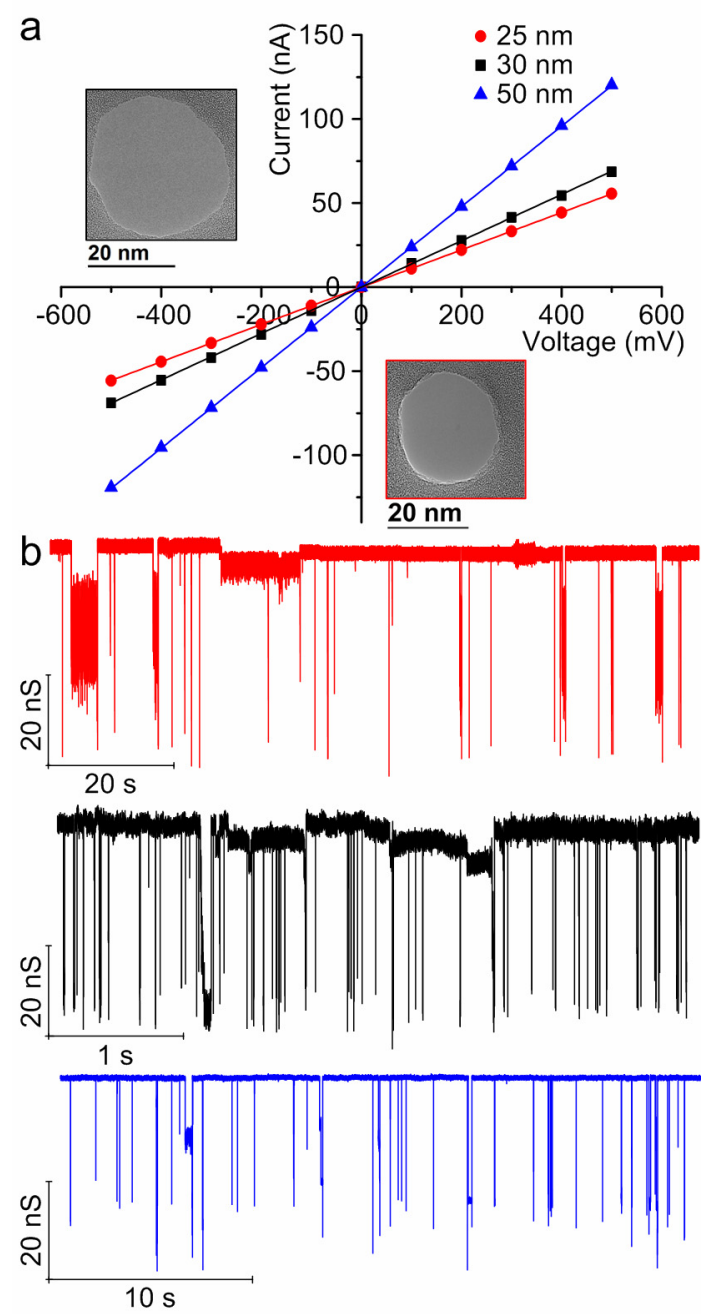

Figure S4. TMV translocation through $25 \mathrm{~nm}, 30 \mathrm{~nm}, 50 \mathrm{~nm}$ nanopores. (a) The current-voltage (I-V) curves of the three pores at $1 \mathrm{M} \mathrm{NaCl}(\mathrm{PH} \mathrm{7.0)}$ ), the insets are TEM images of the $25 \mathrm{~nm}$ (red) and $30 \mathrm{~nm}$ (black) nanopores. (b) Typical conductance traces of three translocation experiments, where the applied voltage of $25 \mathrm{~nm}$ nanopore (red, up) is $500 \mathrm{mV}, 30$ $\mathrm{nm}$ nanopore (black, middle) is $100 \mathrm{mV}$, and $50 \mathrm{~nm}$ nanopore (blue, bottom) is $400 \mathrm{mV}$. 

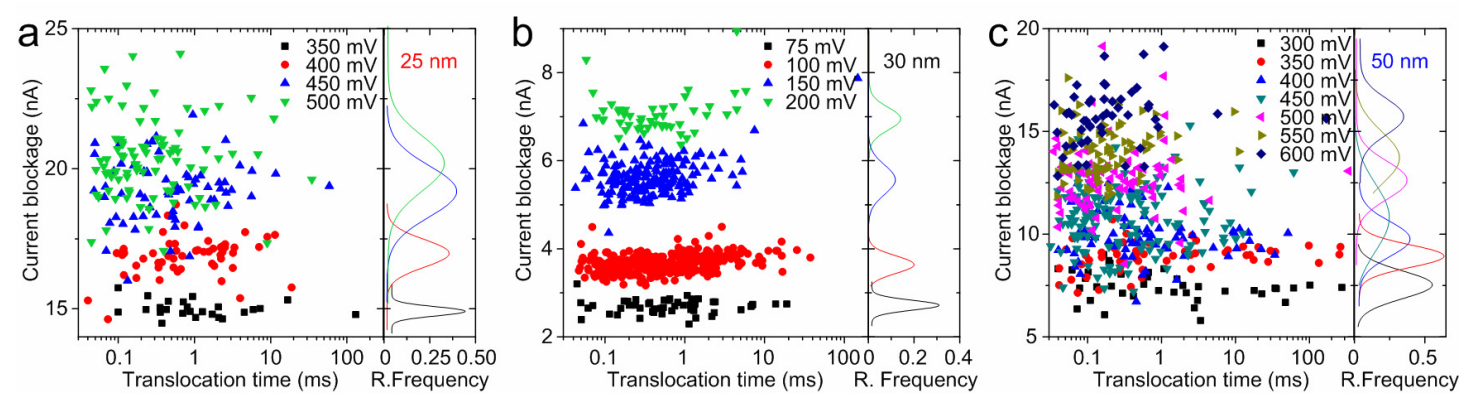

Figure S5. The scatter plots of current blockage $(\Delta I)$ and translocation time $(\Delta t)$ of type $\mathrm{A}$ events of three nanopores. (a) is $25 \mathrm{~nm}$ nanopore, (b) is $30 \mathrm{~nm}$ nanopore, (c) is $50 \mathrm{~nm}$ nanopore.

To avoid the influence of the rotation of TMV (step iii) to the current blockage, only type A events were used to obtain the current blockage of the vertical translocation $\left(\Delta I_{0}\right)$. Figure S5 are the scatter plots of the three nanopores at various voltages, and accompanying distributions are put on the right. The current blockages of the three nanopores as a function of the applied voltage are shown in figure S6. The linear fit with a fixed intercept at 0 shows that Ohm's Law is obeyed and yields the conductance blockage, $\Delta G_{0}=42.4 \mathrm{nS}$ for $25 \mathrm{~nm}$ nanopore, $\Delta G_{0}=35.8 \mathrm{nS}$ for $30 \mathrm{~nm}$ nanopore, $\Delta G_{0}=25.3 \mathrm{nS}$ for $50 \mathrm{~nm}$ nanopore. Note that due to lack of enough type A events in $25 \mathrm{~nm}$ nanopore, type $\mathrm{B}$ and the type $\mathrm{C}$ events with a clear demarcation between the fluctuations due to the rotation to fit in the pore (step iii in figure 2a) and blockade stage (step iv in figure 2a) were included in the plot. The fluctuations at the step iii were cut before fitting by the above equation, as shown in the inset of figure S6.

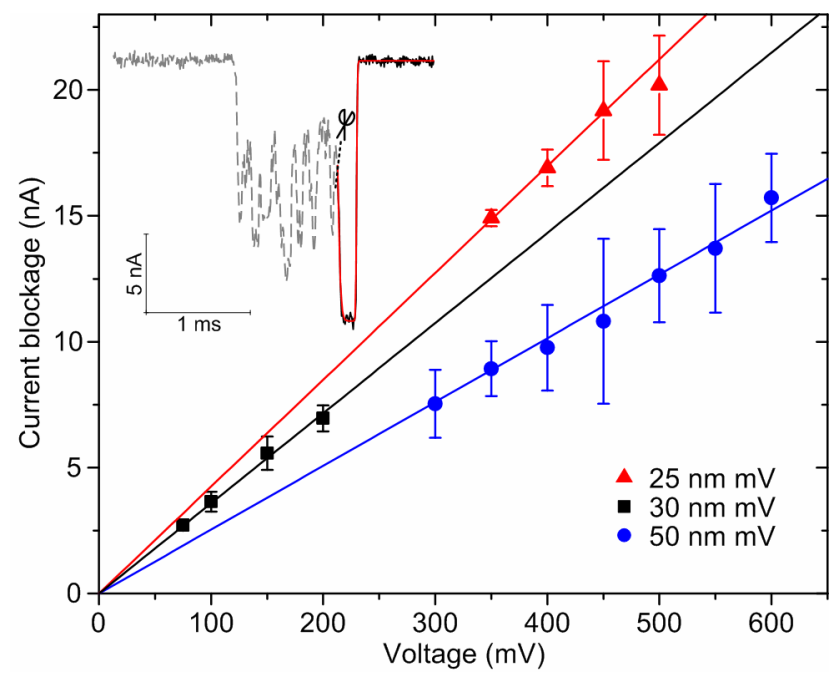

Figure S6. The current blockage of the three nanopores as a function of the applied voltage. The solid lines are linear fits with an intercept at 0 . The inset is a representive type $\mathrm{C}$ event of 25 $\mathrm{nm}$ nanopore at $400 \mathrm{mV}$, showing the fluctuations were cut off before fitting by the sigmoid function to obtain $\Delta I$.

Figure 6 shows the gallery plots of the representive events of TMV translocation though the 
three nanopores, all the events are centered at $0 \mathrm{nS}$. The blue dash line makers the maximum blockage of the access conductance $\left(\Delta G_{\text {acc }}\right)$, where $\Delta G_{\text {acc }}$ is $-12.79 \mathrm{nS}$ for $25 \mathrm{~nm}$ nanopore, -10 $\mathrm{nS}$ for $30 \mathrm{~nm}$ nanopore, $-7.45 \mathrm{nS}$ for $50 \mathrm{~nm}$ nanopore. The red dash line and the green dash line mark the range of the conductance fluctuations during TMV translocation, where the red dash line marks the conductance blockage of the vertical translocation $\left(\Delta G_{0}\right)$, and the green dash line is the maximum conductance blockage $\left(\Delta G_{\max }\right)$ that we recorded.

Figure $\mathrm{S} 7 \mathrm{a}$ and $\mathrm{S} 7 \mathrm{~b}$ plot $\Delta G_{\text {acc }}$ and $\Delta G_{0}$ as a function of the pore diameter respectively. Two major observations can be made. First, the dynamics of the events at $25 \mathrm{~nm}$ and $50 \mathrm{~nm}$ nanopore can be described by the above model as well, which indicates that the appearance of the intriguing dynamics of event in the translocation of TMV is dominated by the high rigidity of TMV, not the pore size. Second, as the pore size increasing, both the access conductance change and the total conductance change of the pore due to a vertical translation decrease.
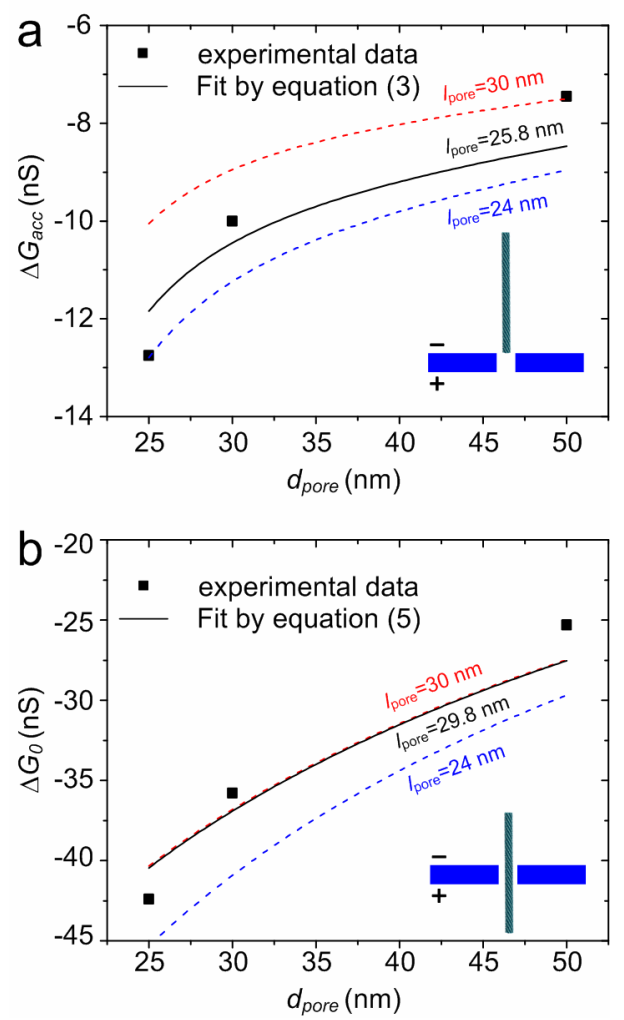

Figure S7. The conductance change of the access region due to the trapping of TMV near the pore entrance and the vertical translocation of TMV as a function of pore diameter. (a) The plot of $\Delta G_{\text {acc }}$ as a function of $d_{\text {pore }}$, and the solid line is a fitting by equation (3), yielding $l_{\text {pore }}=$ $25.8 \mathrm{~nm}$. Two dash lines are the fittings with $l_{\text {pore }}=24 \mathrm{~nm}$ (blue) and $l_{\text {pore }}=30 \mathrm{~nm}$ (red) respectively. (b) $\Delta G_{0}$ as a function of $d_{\text {pore }}$, and the solid line is a fitting by equation (5), yielding $l_{\text {pore }}=29.8 \mathrm{~nm}$. The dash lines are the same as figure S7a. The insets of these two figures are the illustrations of these two cases. 
To give a quantitative analysis, as done by Carlsen et al, ${ }^{20}$ taking account of the access conductance, the total conductance of the pore can be written as ${ }^{3,21}$

$$
G_{\text {TOTAL }}=\left(\frac{1}{G_{\text {pore }}}+\frac{2}{G_{\text {acc }}}\right)^{-1}
$$

Where the access conductance of a nanopore can be written as $G_{a c c}=2 \sigma_{s} d_{\text {pore }}$, and the conductance of the pore without the access region is

$$
G_{\text {pore }}=\frac{\pi d_{\text {pore }}^{2}}{4 l_{\text {pore }}}\left(\sigma_{s}+\frac{4 S \mu_{k}}{d_{\text {pore }}}\right)
$$

Where $\sigma_{s}$ is the conductivity of the solution, $S$ is the surface charge density of the pore wall.

For the case of one end of TMV approaching the access region and trapping near the pore mouth (step ii in figure 2a), the maximum conductance change can be calculated using the following expression ${ }^{20}$

$$
\Delta G_{\mathrm{acc}}=\left(\frac{1}{G_{\mathrm{pore}}}+\frac{1}{G_{\mathrm{acc}}}+\frac{1}{G_{\mathrm{acc}_{T M V}}}\right)^{-1}-G_{T O T A L}
$$

Where the access conductance with TMV occupying the most space of access region (indicating in the insert of figure S7a) can be written as

$$
G_{\mathrm{acc} T M V}=G_{\mathrm{acc}}-G_{T M V_{\mathrm{acc}}}=G_{a c c}-\sigma_{s} \frac{\pi d_{T M V}^{2}}{2 d_{\text {pore }}}
$$

For the case of TMV vertically passing through the pore, as shown in the insert of figure S7b, the conductance change is

$$
\Delta G_{0}=\left(\frac{1}{G_{\text {pore }_{T M V}}}+\frac{2}{G_{\mathrm{acc}_{T M V}}}\right)^{-1}-G_{T_{T O T A L}}
$$

Where the conductance of pore region with TMV vertically occupying the pore is written as ${ }^{22}$

$$
G_{\text {pore }_{T M V}}=G_{\text {pore }}-G_{T M V_{\text {pore }}}=G_{\text {pore }}-\sigma_{s} \frac{\pi d_{T M V}^{2}}{4 l_{\text {pore }}}+\frac{q^{*}}{l_{\text {pore }}} \mu_{k}
$$

The solid black lines in Figure S7a and S7b are the fitting of equation (3) and equation (5) respectively with only one fitting parameter $l_{\text {pore }}$, Where $\sigma_{s}=8.5 \mathrm{~S} / \mathrm{m}$ for $1 \mathrm{M} \mathrm{NaCl}, \mu_{k}$ $=5.2 \times 10^{-8} \mathrm{~m}^{2} / \mathrm{sV}, S=0.03 \mathrm{C} / \mathrm{m}^{2}, 22$ and the effective charge of TMV per unit length $q^{*}$ is 
$2.43 \times 10^{-9} \mathrm{C} / \mathrm{m}$ which is calculated by the $\sigma_{T M V} \times \pi d_{T M V}$, where the surface charge density of TMV $\sigma_{T M V}=0.043 \mathrm{C} / \mathrm{m}^{2} .{ }^{23}$ We obtained $l_{\text {pore }}=25.8 \mathrm{~nm}$ by the fitting of $\Delta G_{\text {acc }}$, and $l_{\text {pore }}=29.8$ $\mathrm{nm}$ by the fitting of $\Delta G_{0}$. For a better comparison, we plot the $\Delta G_{a c c}$ and $\Delta G_{0}$ as a function of the pore diameter at $l_{\text {pore }}=24 \mathrm{~nm}$ (blue) and $l_{\text {pore }}=30 \mathrm{~nm}$ (red), as the dash lines shown in the two figures. In figure $\mathrm{S} 7 \mathrm{a}$, as we used $30 \mathrm{~nm}$ thick silicon nitride membranes, one can find that $\Delta G_{a c c}$ of $50 \mathrm{~nm}$ nanopore is well described by equation (3), while that of the $25 \mathrm{~nm}$ and $30 \mathrm{~nm}$ nanopores are fitted better when $l_{\text {pore }}$ is smaller than $30 \mathrm{~nm}$. The possible reason for this discrepancy is the hour-glass sharp of the pore. ${ }^{21}$ As the $25 \mathrm{~nm}$ and $30 \mathrm{~nm}$ nanopores were fabricated by focused electron beam, the resulting nanopore is of hour-glass shape with a reduced the effective length and bigger openings at the both side of pore. ${ }^{24,25}$ In contrast, the $50 \mathrm{~nm}$ nanopores were drilled by focused $\mathrm{He}^{+}$beam, leading cylindrical shape, and the length of the pore equals to the thickness of the membrane. In figure S7b, the fitting of equation (5) gives $l_{\text {pore }}=29.8 \mathrm{~nm}$ which is consistent with the given membrane thickness $30 \mathrm{~nm}$. 
6. Figure S8. The current traces of TMV translocation through $25 \mathrm{~nm}$ nanopore at 450 $\mathrm{mV}, 400 \mathrm{mV}$, and $350 \mathrm{mV}$.
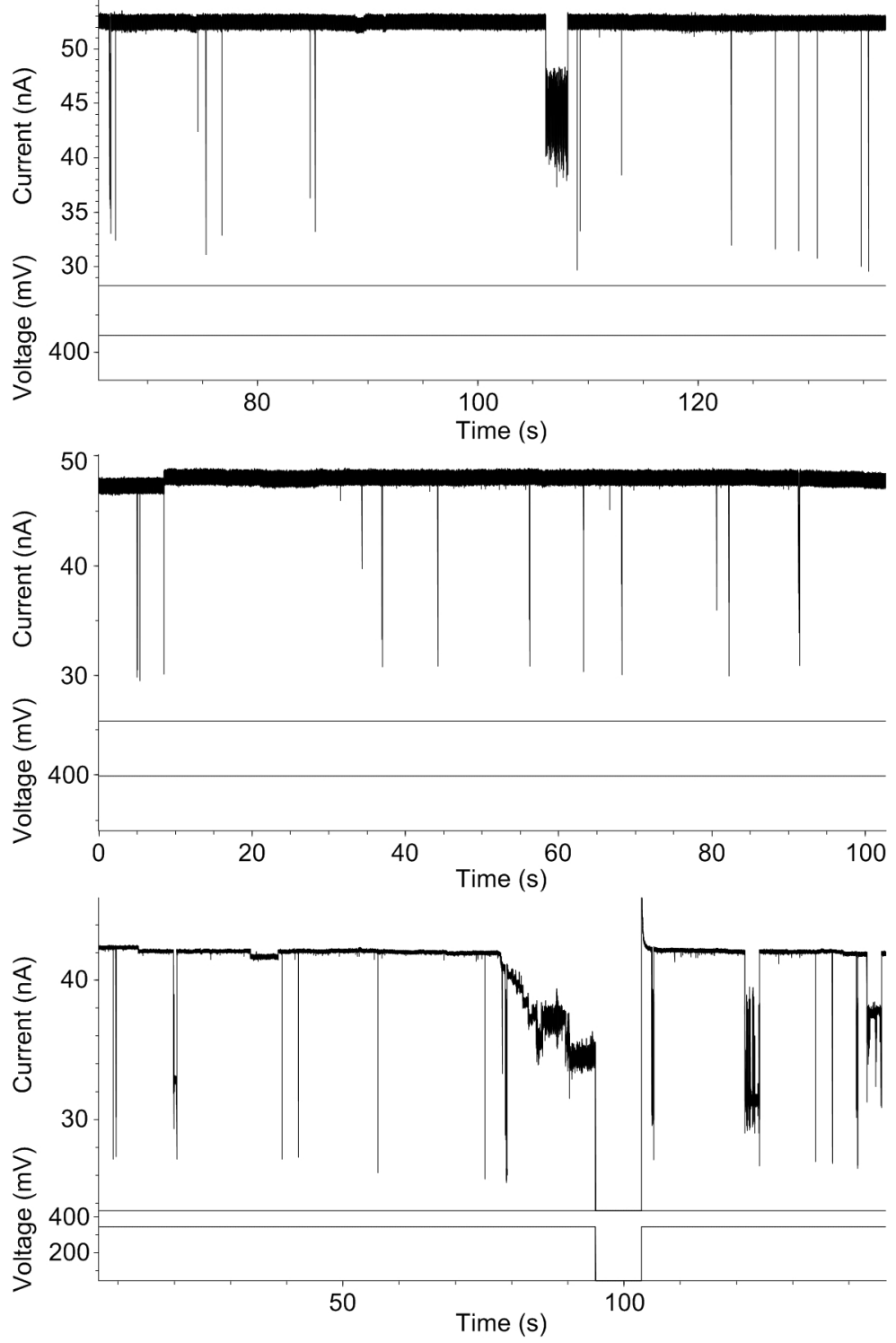
7. Figure S9. The current traces of TMV translocation through $50 \mathrm{~nm}$ nanopore at 200 $\mathrm{mV}, 300 \mathrm{mV}$, and $500 \mathrm{mV}$.
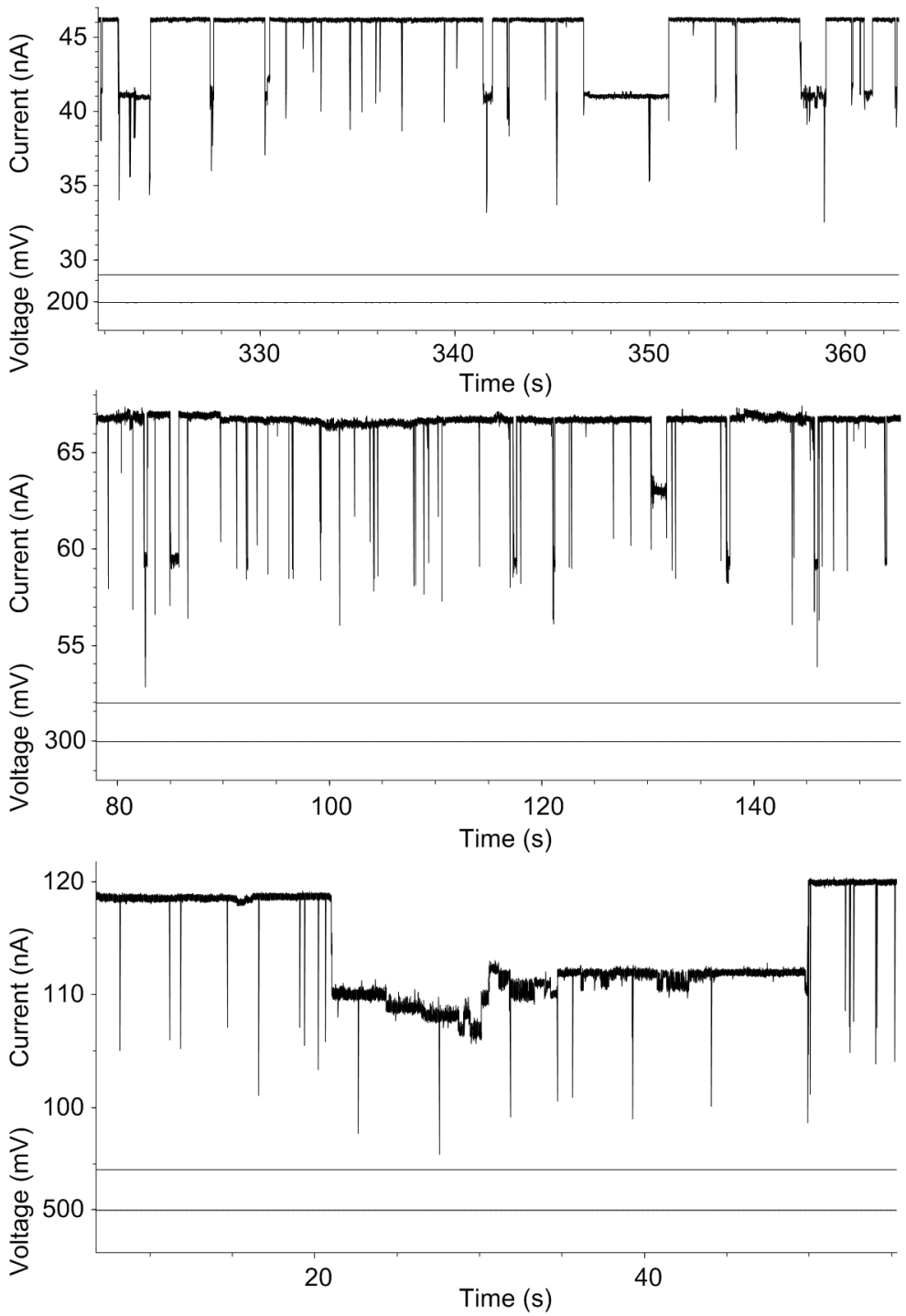


\section{Figure S10. The model and simulated distribution of EOF in a $40 \mathrm{~nm}$ nanopore}

(a) The model size and meshes we used in the simulation, the unit is nm. (b) The color coded distribution of electro-osmosis flow $(v)$ at y direction in $40 \mathrm{~nm}$ nanopore, where TMV approaches an angle of $25^{\circ}$.

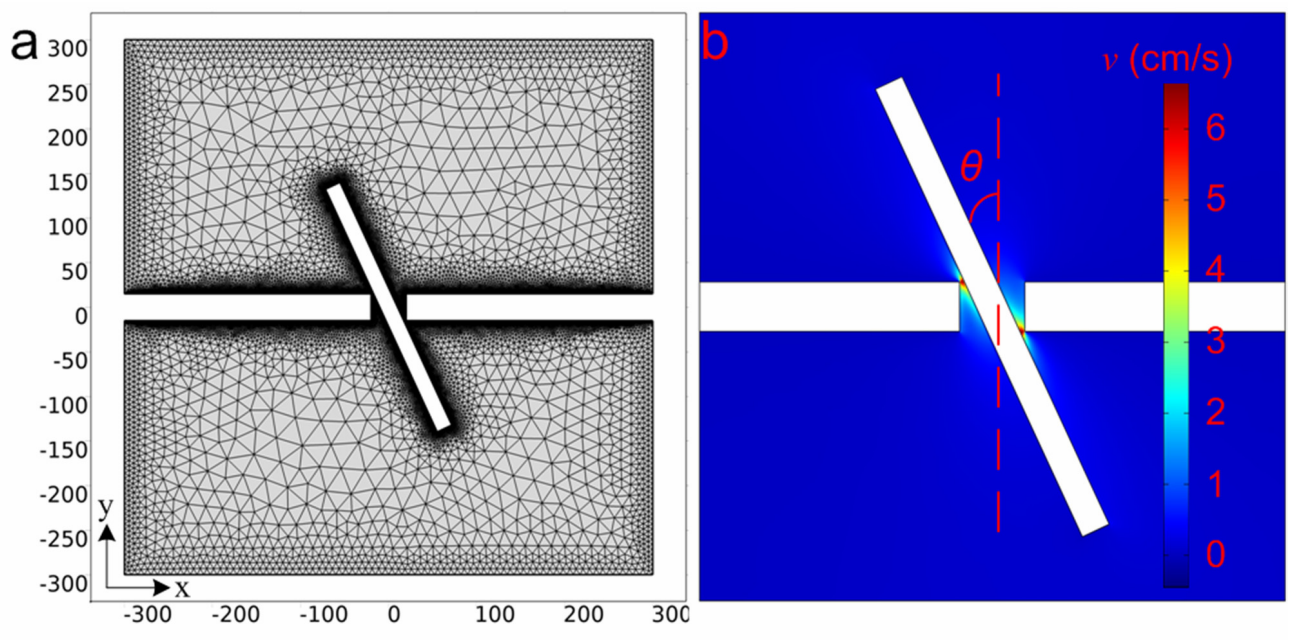


9. Figure S11. The simulated conductance change of the vertical translocation $\left(\Delta G_{0}\right)$ normalized by open-pore conductance $\left(G_{0}\right)$ as a function of pore diameter.

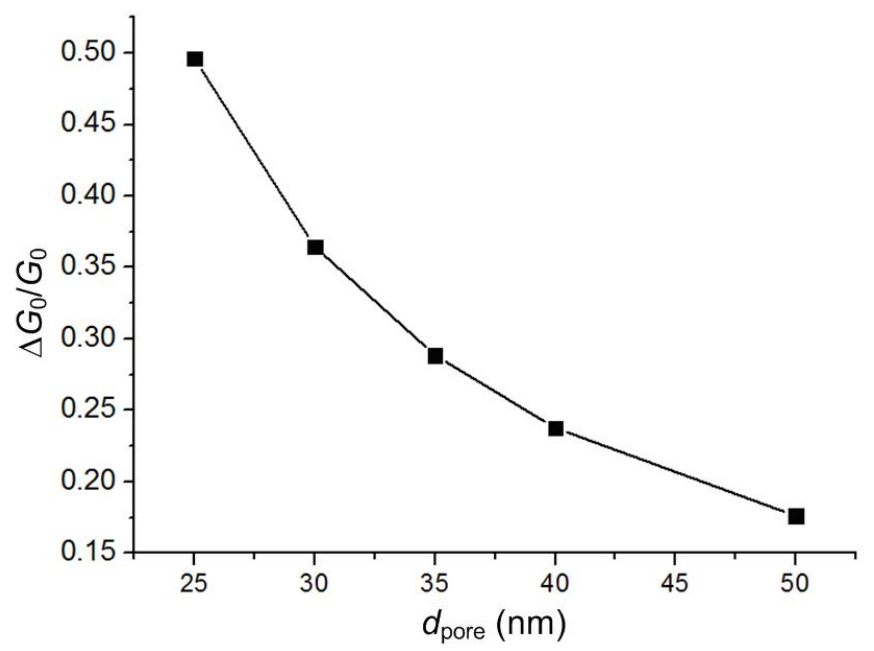


10. Figure S12. The derivation of the equation (2)

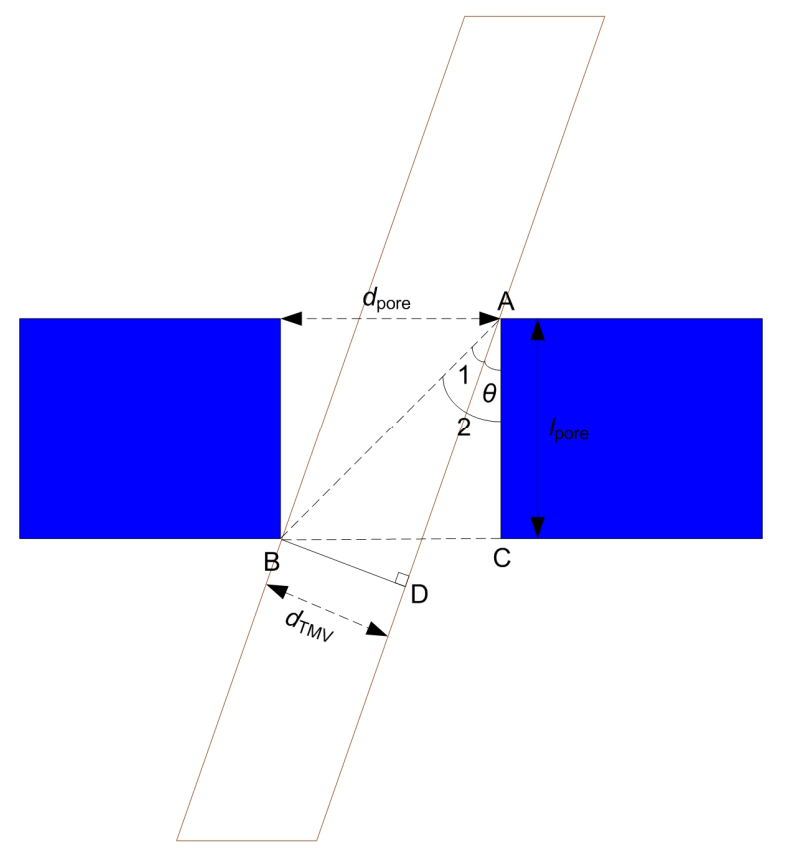

As shown in the above figure where TMV approaches the maximum angle $\theta$, one can get

$$
\angle \theta=\angle 2-\angle 1
$$

Where $\sin \angle 2=B C / A B=d_{\text {pore }} / A B, \sin \angle 1=B D / A B=d_{T M V} / A B$. As $\mathrm{AB}$ can be calculated by $A B=\sqrt{B C^{2}+A C^{2}}=\sqrt{d_{\text {pore }}^{2}+l_{\text {pore }}^{2}}$, we get

$$
\theta_{\max }=\frac{180^{\circ}}{\pi}\left(\arcsin \frac{d_{\text {pore }}}{\sqrt{d_{\text {pore }}^{2}+l_{\text {pore }}^{2}}}-\arcsin \frac{d_{T M V}}{\sqrt{d_{\text {pore }}^{2}+l_{\text {pore }}^{2}}}\right)
$$


11. Figure S13: The scatter plots of $\Delta I_{\max }$ vs $\Delta I_{\text {end }}$ of the three nanopores at all voltages.
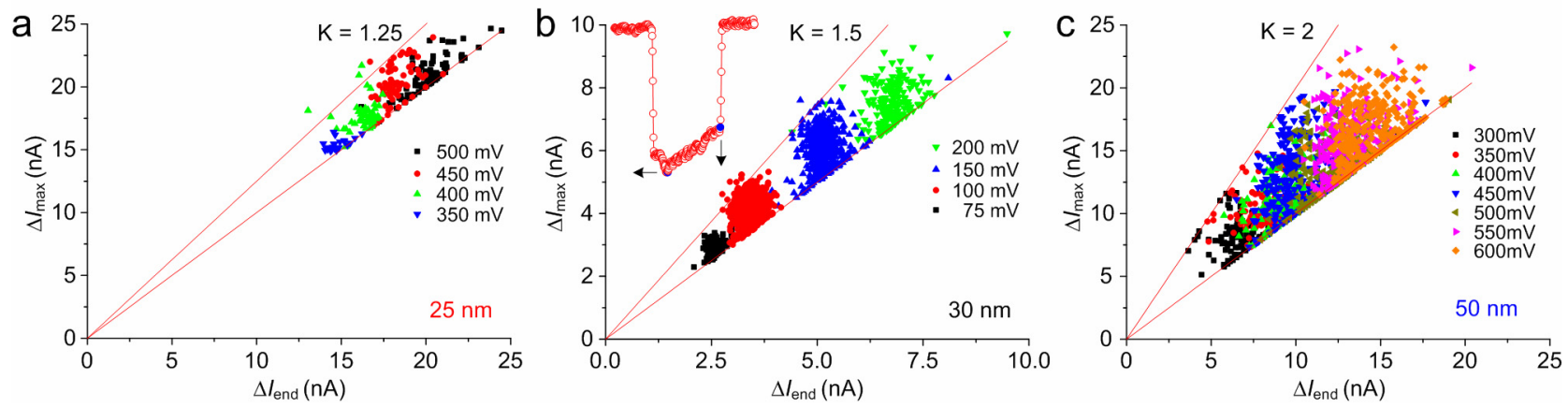

Figure 13. The scatter plots of $\Delta I_{\max } \mathbf{v s} \Delta \boldsymbol{I}_{\text {end }}$ of the three nanopores. (a) $25 \mathrm{~nm}$ nanopore, (b) $30 \mathrm{~nm}$ nanopore, (c) $50 \mathrm{~nm}$ nanopore. The inset of figure $11 \mathrm{~b}$ illustrates how we extracted $\Delta I_{\max }$ and $\Delta I_{\text {end. }}$. The lower red line in each figure is the line of $\Delta I_{\max }=\Delta I_{\text {end }}$, and the up one is $\Delta I_{\max }=\mathrm{K} \Delta I_{\text {end }}$. 


\section{Reference}

(1) Storm, A. J.; Chen, J. H.; Ling, X. S.; Zandbergen, H. W.; Dekker, C. Nat. Mater. 2003, 2, 537-540.

(2) Wu, H.; Liu, H.; Tan, S.; Yu, J.; Zhao, W.; Wang, L.; Liu, Q. The Journal of Physical Chemistry C 2014, 118, 26825-26835.

(3) Wanunu, M.; Dadosh, T.; Ray, V.; Jin, J. M.; McReynolds, L.; Drndic, M. Nature Nanotechnology 2010, $5,807-814$.

(4) Ma, D.; Xie, Y.; Zhang, J.; Ouyang, D.; Yi, L.; Xi, Z. Chem. Commun. 2014, 50, 15581-15584.

(5) McMullen, A.; de Haan, H. W.; Tang, J. X.; Stein, D. Nat. Commun. 2014, 5.

(6) Zhang, K. H.; Luo, K. F. Soft Matter 2013, 9, 2069-2075.

(7) Luo, K.; Ala-Nissila, T.; Ying, S.-C.; Bhattacharya, A. Phys. Rev. Lett. 2007, 99, 148102.

(8) Huopaniemi, I.; Luo, K.; Ala-Nissila, T. J. Chem. Phys. 2006, 125.

(9) He, Y. H.; Tsutsui, M.; Taniguchi, M.; Kawai, T. Journal of Materials Chemistry 2012, 22, 13423-13427.

(10) Allen, M. P.; Tildesley, D. J. Computer simulation of liquids; Oxford University Press, 1987.

(11) Cummins, H. Z.; Carlson, F. D.; Herbert, T. J.; Woods, G. Biophys. J. 1969, 9, 518-546.

(12) Ermak, D. L.; Buckholz, H. Journal of Computational Physics 1980, 35, 169-182.

(13) Liu, H. L.; Pierre-Pierre, N.; Huo, Q. Gold Bulletin 2012, 45, 187-195.

(14) Santos, N. C.; Castanho, M. A. R. B. Biophys. J. 1996, 71, 1641-1650.

(15) Ortega, A.; de la Torre, J. G. J. Chem. Phys. 2003, 119, 9914-9919.

(16) Hu, J.; Wang, P. Y.; Zhao, X.; Lv, L.; Yang, S.; Song, B. A.; Wang, Q. Chem. Commun. 2014, 50, 14125-14128.

(17) Stec, M.; Szatanek, R.; Baj-Krzyworzeka, M.; Baran, J.; Zembala, M.; Barbasz, J.; Waligorska, A.; Dobrucki, J. W.; Mytar, B.; Szczepanik, A.; Siedlar, M.; Drabik, G.; Urbanowicz, B.; Zembala, M. J. Transl. Med. 2015, 13.

(18) Love, A. J.; Makarov, V. V.; Sinitsyna, O. V.; Shaw, J.; Yaminsky, I. V.; Kalinina, N. O.; Taliansky, M. E. Frontiers in Plant Science 2015, 6.

(19) Lee, S. Y.; Culver, J. N.; Harris, M. T. J. Colloid Interface Sci. 2006, 297, 554-560.

(20) Carlsen, A. T.; Zahid, O. K.; Ruzicka, J.; Taylor, E. W.; Hall, A. R. Acs Nano 2014, 8, 4754-4760.

(21) Kowalczyk, S. W.; Grosberg, A. Y.; Rabin, Y.; Dekker, C. Nanotechnology 2011, 22.

(22) Smeets, R. M. M.; Keyser, U. F.; Krapf, D.; Wu, M. Y.; Dekker, N. H.; Dekker, C. Nano Lett. 2006, 6, 89-95.

(23) Ermolina, I.; Morgan, H.; Green, N. G.; Milner, J. J.; Feldman, Y. Biochimica Et Biophysica Acta-General Subjects 2003, 1622, 57-63.

(24) Kim, M. J.; McNally, B.; Murata, K.; Meller, A. Nanotechnology 2007, 18.

(25) Wu, M. Y.; Smeets, R. M. M.; Zandbergen, M.; Ziese, U.; Krapf, D.; Batson, P. E.; Dekker, N. H.; Dekker, C.; Zandbergen, H. W. Nano Lett. 2009, 9, 479-484. 